\title{
Evaluation of multiple laboratory methods in the diagnosis of extrapulmonary tuberculosis
}

\section{Parimala Subramani, Tummalapalli Venkteswarao', Harendra Kumar Malligere Lingaiah², Beena Parvangada Madappa}

Departments of Microbiology and ${ }^{2}$ Pathology, Sri Devaraj Urs Academy of Higher Education and Research, Tamaka, Kolar,

Karnataka, ${ }^{1}$ Department of Microbiology, Travancore Medical College, Kollam, Kerala, India

Address for the Correspondence: Dr. Parimala Subramani, Department of Microbiology, Sri Devaraj Urs Academy of Higher Education and Research, Tamaka, Kolar - 563 101, Karnataka, India. E-mail: mjchand@gmail.com

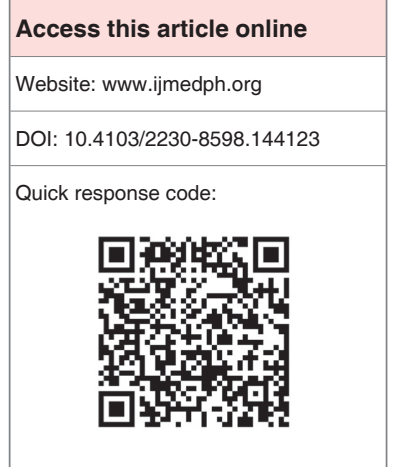

\begin{abstract}
Introduction: Tuberculosis remains a worldwide public health problem. The emergence of human immunodeficiency virus infections (HIV) has further complicated the disease burden as it has rapidly increased the risk of pulmonary and extrapulmonary tuberculosis (EPTB). EPTB is an important clinical entity. The precise diagnosis is very important because early detection of cases and effective treatment if instituted at the right time completely cures the patients of the disease. Objectives: To find out sensitivity and specificity of Ziehl-Neelsens staining (ZN), Auramine staining, and rapid slide culture technique (RSC), comparing them with growth on LowensteinJensens medium (ZN) as the gold standard. Materials and Methods: The present study included 66 clinical specimens from patients suspected of EPTB inclusive of HIV-infected patients. They were subjected to ZN and Fluorescent method of staining, culturing by LJ and RSC method, and comparing growth on LJ medium as the gold standard. Results and Conclusion: Mycobacterium tuberculosis was isolated in 24.2\%. Nontuberculosis Mycobacterium was isolated in $6 \%$ of the 66 samples processed. The highest number of isolation was from lymph node aspirates $(83.3 \%)$. $2 \%$ of the samples were HIV seropositive.
\end{abstract}

Key words: Extrapulmonary tuberculosis, Lowenstein-Jensens, rapid slide culture

\section{INTRODUCTION}

Tuberculosis remains a worldwide public health problem even after 100 years of discovery of Mycobacterium tuberculosis. ${ }^{[1]}$ Approximately 9 million people develop acute tuberculosis every year worldwide. ${ }^{[2]}$ The emergence of human immunodeficiency virus (HIV) infection has further complicated the disease burden as it has rapidly increased the risk of tuberculosis.

Tuberculosis can involve any organ system in the body while the pulmonary tuberculosis (PTB) is the most common presentation. The term extrapulmonary tuberculosis (EPTB) has been used to describe isolated occurrences of tuberculosis at body sites other than the lungs. ${ }^{[3]}$ EPTB constitutes about $15-20 \%$ of all cases of tuberculosis. ${ }^{[3]}$ The clinical presentation of EPTB is atypical. They may present with pyrexia of unknown origin, and this may be the only clinical presentation.

The diagnosis of EPTB is very important because early detection of cases and effective treatment instituted at the appropriate time completely cures the patient. The increase in the incidence of HIV infections has further increased the incidence of EPTB, ${ }^{[4]}$ it is found that among the HIV-TB coinfected patients, EPTB is about $30-70 \%$ more common than PTB. ${ }^{[4]}$ There is no specific, sensitive, inexpensive, and rapid method of diagnosis. Tissue samples for the confirmation of diagnosis can sometimes be difficult to procure. EPTB often presents a diagnostic challenge, most often leading to delay in diagnosis. ${ }^{[5]}$ The study is undertaken to see if different methods of diagnosis can improve the sensitivity of diagnosis of EPTB. Recently, there is renewed interest in rapid slide culture (RSC $)^{[6]}$ which was first used by Sir Robert Koch, though culture on Lowenstein-Jensens (LJ) medium remains the gold standard. RSC is useful for early detection of viable $M$. tuberculosis as the growth occurs within a period of 7 days. $^{[7]}$

\section{Objectives}

1. To find out the sensitivity and specificity of Ziehl-Neelsen's (ZN) staining, Auramine. Staining, and RSC technique and comparing them with growth on LJ medium as the gold standard. 


\section{MATERIALS AND METHODS}

66 clinical specimens which included pleural fluid, pus samples, lymph node aspirates, biopsy specimens, cerebrospinal fluid, synovial fluid, ascitic fluid, urine, and bone marrow aspirates from patients suspected of EPTB at RL Jalappa Hospital and Research Center were collected during the period of December 2008 to August 2010. Patients whose sputum was positive for acid fast bacilli (AFB) were excluded from the study. The specimens collected were sent to the laboratory immediately and were processed, in case of delay these specimens were kept at $4^{\circ} \mathrm{C}$ in the refrigerator after obtaining informed consent. These specimens were examined by $\mathrm{ZN}$ and fluorescent staining (Auramine staining) technique. ${ }^{[7]}$ The samples were further concentrated by modified petroffs method and were cultured on LJ medium ${ }^{[8]}$ and RSC method according to Nair et al. ${ }^{[6]}$

Human blood medium was used for RSC technique. ${ }^{[6]}$ Unused but not $>4$ weeks old citrated human blood was used to prepare the human blood medium (HBM). The blood is diluted with equal volumes of sterile deionized water to cause hemolysis. The medium was made selective by adding trimethoprim $(10 \mathrm{mg} / \mathrm{l})$, amphotericin b $(10 \mathrm{mg} / \mathrm{l})$ and ceftazidime $(100 \mathrm{mg} / \mathrm{l})$. PH of the medium was adjusted between 6.5 and 7.5. Seven $\mathrm{ml}$ of this solution was dispensed in sterile screw capped McCartney bottles with antifungals and antibiotics to eliminate chances of contamination; this constituted one unit of HBM.

Smear was made on the lower one-third of a clean slide and air-dried. The slide was then immersed in the HBM in such way that smear on the slide remained dipped in the medium. Inoculation was done in duplicates. The bottle was incubated at $37^{\circ} \mathrm{C}$ for 7 days. On the $7^{\text {th }}$ day slide was taken out, washed with distilled water and placed in an oven at $80^{\circ} \mathrm{C}$ for $30 \mathrm{~min}$. Any growth was confirmed by $\mathrm{ZN}$ staining and microscopy under oil immersion objective for microcolonies of AFB. A known $M$. tuberculosis strain $\mathrm{H} 37 \mathrm{Rv}$ was used as a positive control and an uninoculated slide as a negative control simultaneously.

Grading of culture by RSC method [Table 1].

The presence of micro colonies was demonstrated by $\mathrm{ZN}$ staining. Growth was graded according to the size of micro colonies [Figure 2].

The growth if any at the end of 12 weeks on LJ culture and by 7 days on RSC was confirmed by Niacin test. Growth on LJ culture [Figure 1] was considered as the gold standard.

\section{RESULTS}

In the present study, a total of 66 patients with clinical suspicion of EPTB was enrolled. 38 (57.5\%) were males and $28(42.5 \%)$ were females. Majority belonged to the age group of 21-30 years. Most of the samples were obtained from cases of pleural effusion (43.9\%) followed by pus ( $16.6 \%$ ), from parietal wall abscess, scrotal ulcer, psoas abscess, port site abscess following cholecystectomy, breast abscess, chronic suppurative otitis media, and lymph node aspirates $(9.1 \%)$.
Of the 66 specimens processed, 22 were positive for AFB either by ZN stain, Auramine staining, LJ culture or RSC. 16 were identified as $M$. tuberculosis [Table 2] and 4 were nontuberculous Mycobacterium which included Mycobacterium chelonae and Mycobacterium fortuitum by culture and two were positive by staining methods only. Majority of the isolates were from lymph node aspirates $5(83.3 \%)$. The percentage of positivity with $\mathrm{ZN}$ and fluorescent staining technique was $27.2 \%$. The isolation rate by LJ culture was $30.4 \%$, and RSC was $24.2 \% .2(3 \%)$ samples which were positive by $\mathrm{ZN}$ staining, fluorescent staining methods, but were negative by LJ culture and RSC.

\section{Table 1: Grading of RSC method ${ }^{[16]}$}

\begin{tabular}{|c|c|c|c|}
\hline \multicolumn{3}{|l|}{ ZN staining } & Grading \\
\hline \multicolumn{3}{|c|}{$\begin{array}{l}\text { No multiplication of AFB as compared with an } \\
\text { un-incubated control }\end{array}$} & 0 \\
\hline \multicolumn{3}{|c|}{ Small clumps of up to four bacilli } & $1+$ \\
\hline \multicolumn{3}{|c|}{ Large clumps of bacilli, but no cord formation } & $2+$ \\
\hline \multicolumn{3}{|c|}{ Microcolonies with some cord formation } & $3+$ \\
\hline \multicolumn{3}{|c|}{ Large micro colonies with good cord formation } & $4+$ \\
\hline \multicolumn{4}{|c|}{ RSC = Rapid slide culture, ZN = Ziehl-Neelsens, AFB = Acid fast bacilli } \\
\hline \multicolumn{4}{|c|}{$\begin{array}{l}\text { Table 2: Isolation of Mycobacterium tuberculosis } \\
\text { from different sites }\end{array}$} \\
\hline Sample & $\begin{array}{c}\text { Number } \\
\text { of sample } \\
\text { processed }\end{array}$ & $\begin{array}{c}\text { Total } \\
\text { positive } \\
(\%)\end{array}$ & Percentage \\
\hline $\begin{array}{l}\text { Lymph node } \\
\text { aspirate }\end{array}$ & 6 & 5 & 83.3 \\
\hline Pleural fluid & 29 & 4 & 13.8 \\
\hline Pus & 11 & 3 & 27.8 \\
\hline Synovial fluid & 3 & 1 & 33.3 \\
\hline Urine & 2 & 1 & 50.0 \\
\hline Ascitic fluid & 3 & 1 & 33.3 \\
\hline $\begin{array}{l}\text { Biopsy } \\
\text { specimens }\end{array}$ & 6 & 1 & 16.7 \\
\hline CSF & 4 & 0 & 0.0 \\
\hline $\begin{array}{l}\text { Bone marrow } \\
\text { aspirate }\end{array}$ & 2 & 0 & 0.0 \\
\hline Total & 66 & $16(24.2)$ & \\
\hline
\end{tabular}

CSF = Cerebrospinal fluid

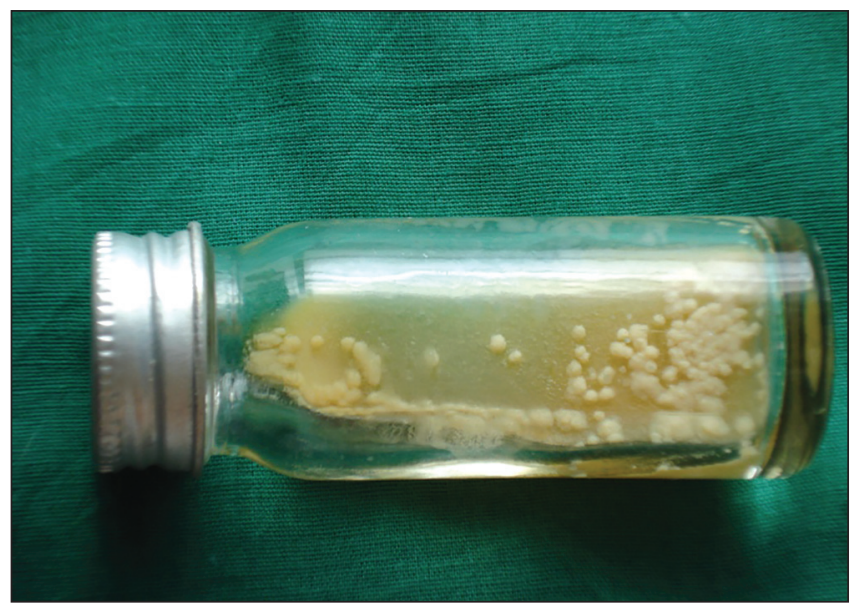

Figure 1: Growth of Mycobacterium tuberculosis on lowensteinJensens medium 


\section{DISCUSSION}

In the present study, there was male preponderance, with a maximum number of patients in the age group of 21-30 years. Our study showed a positivity of $22(33.3 \%)$ by one or more methods of staining and culture. Narang et al..$^{[9]}$ showed a positivity of $35.94 \%$ considering staining technique by ZN method, LJ culture and histopathological examination in lymph node specimens only. Maximum number of isolation of M. tuberculosis was from lymph node aspirates (83.3\%). Vanajakumar et al. ${ }^{[10]}$ has reported $98 \%$ isolation from lymph node specimens. There was no significant difference observed between the results obtained by $\mathrm{ZN}$ and fluorescent method of staining. However, fluorescent staining has offered the advantage of screening the smears under low power where large numbers of slides are screened in less time reducing observers fatigue as quoted in the bulletin of ICMR. ${ }^{[1]}$ RSC had sensitivity of $80 \%$, specificity of $100 \%$, and positive predictive value (PPV) of $100 \%$, and negative predictive value of $92 \%$ [Table 3]. It had sensitivity comparable with smear microscopy. It has the advantage of demonstrating microcolonies by light microscopy in 7 days.

To the best of our search to date, there are only three studies on RSC technique from India. Purohit et al..$^{[12]}$ evaluated a new medium sheep blood medium and HBM and found that the results between the two were equally good. ${ }^{[12]}$

The newer methods in the detection of $M$. tuberculosis are Bactec system, mycobacterial growth indicator tube, and molecular methods which are rapid and effective. These tests are very expensive and technically demanding and beyond the reach of many laboratories in India. One important issue of concern in India is affordability, as we have a larger population who are poor. ${ }^{[13]}$

Rapid slide culture, when compared to the above methods, is rapid, simple, and affordable and the turnaround time for detection of M. tuberculosis is 7 days which proves it being more advantageous than LJ culture. ${ }^{[14]}$

Jena et al..$^{[15]}$ in 1995 and George et al. ${ }^{[16]}$ in 1998 used HBM for drug susceptibility testing in pulmonary samples. Jena et al. ${ }^{[15]}$ compared it with conventional LJ culture in fresh untreated cases of PTB. A positivity of $65.2 \%$ for RSC and $85.1 \%$ for LJ culture was shown in their study. ${ }^{[15]}$

In our study, we used the RSC method as a novel method for isolation of $M$. tuberculosis in extra-pulmonary specimens. The isolation rate was $24.2 \%$ in RSC and $30.4 \%$ in LJ culture showing slightly higher percentage of isolation on LJ culture. Jena et al. found RSC to be more sensitive than smear microscopy. ${ }^{[15]}$ Nair et al. ${ }^{[6]}$ found smear microscopy to be more sensitive than RSC. In our study, RSC had a sensitivity of $80 \%$ comparable with the sensitivity of smear microscopy. A PPV of $100 \%$ indicates that the diagnostic potential of the test is good.

It was found that $4(6 \%)$ of the specimens were smear negative and culture positive [Table 4]. EPTB is paucibacillary in nature and hence most often they are not detected by smear microscopy. This can be explained because of less number of organisms present in the sample as, there must be at least $10^{4}$ organisms in the sample to be detected by smear microscopy whereas the number of organisms required for culture to be positive is $10-100$ organisms per ml. ${ }^{[17,18]}$

In the present study, $2(3 \%)$ were smear positive and culture negative [Table 5]. Aparna et al. have shown $12.1 \%$ to be smear positive and culture negative in lymph node aspirates. ${ }^{[19]}$

This could be attributed to treatment with anti-tubercular drugs and broad spectrum antibiotics such as amoxicillin, fluoroquinolones reported to be inhibitory to $M$. tuberculosis that might lead to negative culture and positive smear. Further clinical history revealed that they were treated with levofloxacin for 2 weeks.

\section{CONCLUSION}

From the above findings, it can be concluded that RSC is as good as LJ culture. It is a rapid, cheap, and effective method for obtaining culture confirmation of tuberculosis and considered most suitable in a country like India where tuberculosis is rampant. The need of the hour being rapid detection of $M$. tuberculosis and prompt treatment. However, LJ medium still remains the gold standard.

\begin{tabular}{|c|c|c|c|c|}
\hline Methods & Sensitivity & Specificity & PPV & NPV \\
\hline Direct smear & 80 & 95.65 & 88.89 & 91.67 \\
\hline Fluorescent stain & 80 & 95.65 & 88.89 & 91.67 \\
\hline RSC & 80 & 100 & 100 & 92 \\
\hline
\end{tabular}

PPV = Positive predictive value, NPV = Negative predictive value, $\mathrm{RSC}=$ Rapid slide culture, LJ = Lowenstein-Jensens

\begin{tabular}{|c|c|c|c|c|}
\hline \multirow[t]{2}{*}{ Specimens } & \multicolumn{2}{|c|}{ Staining methods } & \multicolumn{2}{|c|}{ Culture methods } \\
\hline & $\overline{Z \mathbf{N}}$ & Fluorescent & 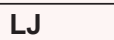 & RSC \\
\hline Pleural fluid & Negative & Negative & Positive & Positive \\
\hline Ascitic fluid & Negative & Negative & Positive & Positive \\
\hline $\begin{array}{l}\text { Endometrial } \\
\text { biopsy }\end{array}$ & Negative & Negative & Positive & Positive \\
\hline $\begin{array}{l}\text { Pus shoulder } \\
\text { joint }\end{array}$ & Negative & Negative & Positive & Positive \\
\hline
\end{tabular}

Table 5: Specimens which were smear positive
and culture negative
\begin{tabular}{llllll} 
Specimen & \multicolumn{2}{l}{ Staining methods } & & Culture methods \\
\cline { 2 - 2 } & ZN & Fluorescent & & LJ & RSC \\
\hline $\begin{array}{l}\text { Bone marrow } \\
\text { aspirate }\end{array}$ & Positive & Positive & & Negative & Negative \\
FNAC ileocecal & Positive & Positive & & Negative & Negative
\end{tabular}

region

RSC = Rapid slide culture, LJ = Lowenstein-Jensens, ZN = Ziehl-Neelsens, FNAC = Fine needle aspiration cytology 


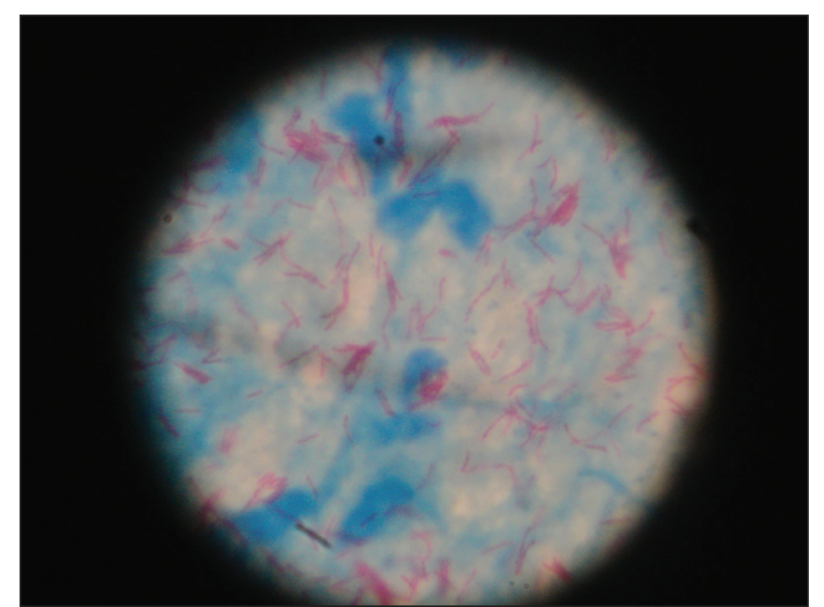

Figure 2: Zeil-Neelsens staining: Rapid slide culture showing microcolonies with some cord formation (grade-3+)

In the present study, HBM was used in RSC, blood was screened for all the pathogens associated with biohazard. However, an alternative like fetal calf serum can replace blood, and RSC can be used as a diagnostic test. It is useful in early confirmation of $M$. tuberculosis which makes it an ideal diagnostic test in a country like India where tuberculosis is rampant. However, since the sample size is small, a better conclusion can be derived by conducting a study with a larger number of samples.

\section{REFERENCES}

1. Park K. Epidemiology of communicable diseases. In: Park's Text Book of Preventive and Social Medicine. $17^{\text {th }}$ ed. Jabalpur: Banarsidas Bhanot; 2006. p. 115-268.

2. World Health Organisation. Global Tuberculosis Control: A Short Update to the 2009 Report. 2009.

3. Sharma SK, Mohan A. Extrapulmonary tuberculosis. Indian J Med Res 2004;120:316-53.

4. Arora VK, Chopra KK. Extra pulmonary tuberculosis. Indian J Tuberc 2007;54:165-7.

5. Gonzalez OY, Adams G, Teeter LD, Bui TT, Musser JM, Graviss EA. Extra-pulmonary manifestations in a large metropolitan area with a low incidence of tuberculosis. Int J Tuberc Lung Dis 2003;7:1178-85.
6. Nair L, Sudarsana J, Nizamuddin, Karim S, Kumar S. Preliminary report on rapid slide culture of Mycobacterium tuberculosis. JAcad Clin Microbiol 1998;1:151-3.

7. Manual on Isolation, Identification and Sensitivity testing of Mycobacterium tuberculosis. $2^{\text {nd }}$ ed. Bangalore: National Tuberculosis Institute, Director General of Health Services, Government of India; 1998.

8. Koneman EW, Allen SD, Janda WM, Schreckenbeger PC, Winn WC. In: Koneman's Color Atlas and Textbook of Diagnostic Microbiology. $4^{\text {th }}$ ed. Philadelphia: JB Lippincott; 2006. p. 711.

9. Narang P, Narang R, Mendiratta DK, Sharma SM, Narang R, Nayar S. Field study to evaluate the bacteriological parameters in the diagnosis of lymph node tuberculosis in children. Indian J Tuberc 1998;45:211.

10. Vanajakumar, Selvakumar N, Jawahar MS, Rajaram K, Paramasivan CN. Transportation of lymph node biopsy specimens in selective Kirchner's liquid medium for culture of tubercle bacilli. J Med Microbiol 1997;46:260-2.

11. ICMR Bulletin. New Delhi. Vol. 32. 2002. p. 8.

12. Purohit SD, Gupta ML, Chauhan A, Nanavati V. A new medium for rapid slide culture of tubercle bacilli. Indian J Pathol Microbiol 1993;36:370-5.

13. Katoch VM. Newer diagnostic techniques for tuberculosis. Indian J Med Res 2004;120:418-28.

14. Sanjeev H, Karnaker VK, Rekha R, Pai Asha KB, Ganesh HR, Krishnaprasad HR. Rapid slide culture: Relevance to the modern day diagnosis of tuberculosis. J Clin Diagn Res 2012;6:378-81.

15. Jena J, Nema SK, Panda BN, Rajan KE. Comparative efficacy of rapid slide culture of $M$. tuberculosis and conventional LJ medium culture in diagnosis and management of pulmonary tuberculosis. Indian J Tuberc 1995;42:151-4.

16. George K, Nair L, Sudarshana J. Rapid drug susceptibility test for M. tuberculosis by slide culture. J Acad Clin Microbiol 2008;10:5-13.

17. Borriello SP, Murray PR, Funkeg. Mycobacterium tuberculosis complex, Mycobacterium leprae; and others slow growing Mycobacterim. Bacteriology. In: Topley, Wilson's, Microbiology and Microbial Infection. $10^{\text {th }}$ ed., Vol. 2. Washington DC: Hodaer Aevol D; 2005. p. 118.

18. Watt B, Rayner A, Harris G. Mycobacterium. In: Collee JG, Fraser AG, Marmion BP, Simmons A, editors. Mackie and McCartney Practical Medical Microbiology. $14^{\text {th }}$ ed. New York: Churchill Livingstone; 1996. p. 329-41.

19. Kishore Reddy VC, Aparna S, Prasad CE, Srinivas A, Triveni B, Gokhale S, et al. Mycobacterial culture of fine needle aspirate - A useful tool in diagnosing tuberculous lymphadenitis. Indian $\mathrm{J}$ Med Microbiol 2008;26:259-61.

How to cite this article: Subramani P, Venkteswarao T, Malligere Lingaiah HK, Madappa BP. Evaluation of multiple laboratory methods in the diagnosis of extrapulmonary tuberculosis. Int J Med Public Health 2014;4:482-5.

Source of Support: Nil, Conflict of Interest: None declared. 